\title{
Correlations derived from modern nucleon-nucleon potentials
}

\author{
H. Müther \\ Institut für Theoretische Physik, Universität Tübingen, D-72076 Tübingen, Germany
}

A. Polls

Departament d'Estructura i Costituents de la Matèria, Universitat de Barcelona, E-08028 Barcelona, Spain

(Received 2 August 1999; published 16 December 1999)

\begin{abstract}
Various modern nucleon-nucleon $(N N)$ potentials yield a very accurate fit to the nucleon-nucleon scattering phase shifts. The differences between these interactions in describing properties of nuclear matter are investigated. Various contributions to the total energy are evaluated employing the Hellmann-Feynman theorem. Special attention is paid to the two-nucleon correlation functions derived from these interactions. Differences in the predictions of the various interactions can be traced back to the inclusion of nonlocal terms.
\end{abstract}

PACS number(s): 21.65. $+\mathrm{f}$

\section{INTRODUCTION}

The microscopic theory of nuclear structure based on realistic nucleon-nucleon $(N N)$ interactions is a very demanding subject because it requires the description of a strongly correlated many-fermion system. Attempts to determine, e.g., the energy of nuclei from a realistic $N N$ interaction by using the mean-field or Hartree-Fock approximation fail badly: such attempts typically yield unbound nuclei. The strong short-range and tensor components of a realistic $N N$ interaction induce correlations into the many-body wave function of nuclear systems. Many attempts have been made to measure these correlations in detail. As an example for such measurements, we mention the exclusive $\left(e, e^{\prime} N N\right)$ reactions, which have been made possible using modern electron accelerators $[1,2]$. The hope is that the detailed analysis of such experiments yields information about the correlated wave function of the nucleon pair absorbing the virtual photon. This could be a very valuable test for the model of the $N N$ interaction producing these correlations.

In recent years, a new generation of realistic $N N$ potentials has been developed which produce very accurate fits of the proton-proton and proton-neutron scattering phase shifts [3-5]. Since these fits are based on the same phase-shift analysis by the Nijmegen group [6] and yield a value for the $\chi^{2} /$ datum very close to one, these various potentials could be called phase-shift equivalent $N N$ interactions.

This means that the on-shell matrix elements for the transition matrix $T$ are essentially identical. This, however, does not imply that the underlying potentials nor the effective interaction between off-shell nucleons moving inside a nucleus are the same. Indeed it has been demonstrated that these phase-shift equivalent potentials yield different results even for the deuteron. Of course all of them reproduce the same empirical binding energy and other observables, because these are part of the observables to which the interaction has been fitted. However, the various contributions to the total energy, the kinetic energy, and the potential energy in the ${ }^{3} S_{1}$ and ${ }^{3} D_{1}$ partial waves, are quite different indicating that also the two-body wave functions must be different $[7,8]$.

It is one of the aims of this study to explore whether similar differences can also be observed in calculating the energy of nuclear matter. These energies are calculated using the Brueckner-Hartree-Fock (BHF) approximation. The BHF approach for nuclear matter assumes a model wave function of a free Fermi gas, occupying plane-wave states up to the Fermi momentum. The effects of correlations are taken into account in terms of the Brueckner $G$ matrix. The BHF approximation does not give direct access to quantities like the kinetic or the potential energy. However, in the next section we will illustrate how the Hellman-Feynman theorem [9] can be used to calculate these quantities and also the expectation value of the $\pi$ exchange calculated for the correlated wave function. The results for various contributions to the binding energy as well as the wave functions of correlated $N N$ pairs in nuclear matter will be presented in Sec. III. The last section contains a summary and conclusions.

\section{CORRELATIONS IN THE BRUECKNER-HARTREE- FOCK APPROXIMATION}

The central equation of the BHF approximation is the Bethe-Goldstone equation, which defines an effective interaction $G$ for two nucleons in nuclear matter occupying the plane wave states $i$ and $j$ by

$$
G|i j\rangle=V|i j\rangle+V \frac{Q}{\epsilon_{i}+\epsilon_{j}-H_{0}} G|i j\rangle .
$$

Here and in the following $V$ stands for the bare $N N$ interaction, $Q$ denotes the Pauli operator, which prevents the interacting nucleons from going into intermediate states with momenta below the Fermi momentum $k_{F}, H_{0}$ defines the spectrum of intermediate two-particle states, and the BHF single-particle energies are defined by

$$
\boldsymbol{\epsilon}_{i}=\frac{\hbar^{2} k_{i}^{2}}{2 m}+\int_{0}^{k_{F}} d^{3} k_{j}\langle i j|G| i j\rangle,
$$

as the sum of the kinetic energy of a free nucleon with mass $m$ and momentum $k_{i}$ and the potential energy. The singleparticle potential corresponds to the Hartree-Fock approximation but calculated in terms of the effective interaction $G$ rather than the bare interaction $V$. Also the total energy of the 
system is calculated in a similar way containing the kinetic energy per nucleon of a free Fermi gas

$$
\frac{T_{\mathrm{FG}}}{A}=\frac{3}{5} \frac{\hbar^{2} k_{F}^{2}}{2 m},
$$

and the potential energy calculated in the Hartree-Fock approximation replacing $V$ by the effective interaction $G$ (For a more detailed description see, e.g., [10].) This means that the BHF approach considers a model wave function, which is just the uncorrelated wave function of a free Fermi gas and all information about correlations are hidden in the effective interaction $G$. Since this effective interaction is constructed such that $G$ applied to the uncorrelated two-body wave function yields the same result as the bare interaction $V$ acting on the correlated wave function

$$
G|i j\rangle=V|i j\rangle_{\mathrm{corr}}
$$

the comparison of this equation with Eq. (1) allows the definition of the correlated two-nucleon wave function as

$$
|i j\rangle_{\mathrm{corr}}=|i j\rangle+\frac{Q}{\epsilon_{i}+\epsilon_{j}-H_{0}} G|i j\rangle .
$$

This representation demonstrates that the correlated wave function contains the uncorrelated one plus the so-called defect function, which in this approach should drop to zero for relative distances between the two nucleons, which are larger than the healing distance.

The BHF approach yields the total energy of the system including effects of correlations. Since, however, it does not provide the correlated many-body wave function, one does not obtain any information about, e.g., the expectation value for the kinetic energy using this correlated many-body state. To obtain such information one can use the HellmannFeynman theorem, which may be formulated as follows: Assume that one splits the total Hamiltonian into

$$
H=H_{0}+\Delta V
$$

and defines a Hamiltonian depending on a parameter $\lambda$ by

$$
H(\lambda)=H_{0}+\lambda \Delta V
$$

If $E_{\lambda}$ defines the eigenvalue of

$$
H(\lambda)\left|\Psi_{\lambda}\right\rangle=E_{\lambda}\left|\Psi_{\lambda}\right\rangle,
$$

the expectation value of $\Delta V$ calculated for the eigenstates of the original Hamiltonian $H=H(1)$ is given as

$$
\langle\Psi|\Delta V| \Psi\rangle=\left.\frac{\partial E_{\lambda}}{\partial \lambda}\right|_{\lambda=1} .
$$

The BHF approximation can be used to evaluate the energies $E_{\lambda}$, which also leads to the expectation value $\langle\Psi|\Delta V| \Psi\rangle$ employing Eq. (9). In the present work we are going to apply the Hellmann-Feynman theorem to determine the expectation value of the one-pion-exchange term $\Delta V=V_{\pi}$ contained in the different interactions. Furthermore, we also calculate the expectation value for total $N N$ interaction $\langle\Psi|V| \Psi\rangle$ in the same way. From this expectation value and the total energy per nucleon $E$, which is also calculated in the BHF approximation, we can determine the expectation value for the kinetic energy as $\langle T\rangle=E-\langle\Psi|V| \Psi\rangle$.

\section{RESULTS AND DISCUSSION}

The main aim of the work presented here is to investigate differences in nuclear structure calculations originating from four different realistic $N N$ interactions, which are phase-shift equivalent. These four interactions are the so-called chargedependent Bonn potential (CD-Bonn) [5], the Argonne V18 (ArV18) [4] and versions I (Nijm1) and II (Nijm2) of the Nijmegen interaction [3]. All these models for the $N N$ interaction include a one-pion exchange (OPE) term, using essentially the same $\pi N N$ coupling constant, and account for the difference between the masses of the charged $\left(\pi_{ \pm}\right)$and neutral $\left(\pi_{0}\right)$ pion. However, even this long-range part of the $N N$ interaction, which is believed to be well understood, is treated quite differently in these models. The Nijmegen and the Argonne V18 potentials use the local approximation, while the pion contribution to the CD-Bonn potential is derived in a relativistic framework assuming pseudoscalar coupling. It has recently been shown that the nonlocalities included in the relativistic description of the CD-Bonn potential tends to lead to smaller $D$-state probabilities in the deuteron [8].

The description of the short-range part is also different in these models. The $N N$ potential Nijm2 [3] is a purely local potential in the sense that it uses the local form of the OPE potential for the long-range part and parametrizes the contributions of medium and short-range distances in terms of local functions (depending only on the relative displacement between the two interacting nucleons) multiplied by a set of spin-isospin operators. The same is true for the Argonne $V_{18}$ potential [4]. The $N N$ potential denoted by Nijm1 [3] uses also the local form of OPE but includes a $\mathbf{p}^{2}$ term in the medium- and short-range central force [see Eq. (13) of Ref. [3]] which may be interpreted as a nonlocal contribution to the central force. The CD-Bonn is derived in the framework of the relativistic meson field theory. It is calculated in momentum space and contains nonlocal terms in the short-range as well as long-range part including the pion-exchange contribution.

First differences in the prediction of nuclear properties obtained from these interactions are displayed in Table I which contains various expectation values calculated for nuclear matter at the empirical saturation density, which corresponds to a Fermi momentum $k_{F}$ of $1.36 \mathrm{fm}^{-1}$. The most striking indication for the importance of nuclear correlations beyond the mean-field approximation may be obtained from the comparison of the energy per nucleon calculated in the mean-field or Hartree-Fock (HF) approximation. All energies per nucleon calculated in the (HF) approximation are positive, therefore, far away from the empirical value of -16 $\mathrm{MeV}$. Only after inclusion of $N N$ correlations in the BHF approximation results are obtained which are close to the experiment. While the $\mathrm{HF}$ energies range from $4.6 \mathrm{MeV}$ in 
TABLE I. Energies calculated for nuclear matter with Fermi momentum $k_{F}=1.36 \mathrm{fm}^{-1}$. Results are listed for the energy per nucleon calculated in BHF $(\langle E\rangle)$ and the Hartree-Fock $\left(\langle E\rangle_{\mathrm{HF}}\right)$ approximation. Furthermore the expectation value for the $N N$ interaction $\langle V\rangle$, the kinetic energy $\left\langle T_{\mathrm{Kin}}\right\rangle$, and the one-pionexchange term $\left\langle V_{\pi}\right\rangle$ are listed. For completeness we also give the $D$-state probability calculated for the deuteron $P_{D}$. Results are presented for the charge-dependent Bonn (CD-Bonn) [5], the Argonne V18 (ArV18) [4], and two Nijmegen (Nijm1, Nijm2) [3] interactions. For a comparison results are also given for three older versions of the Bonn interaction $(A, B, C)$ [11] and the Reid soft-core potential [12], which is supplemented in partial waves in which it is not defined by the OBE $C$ potential. All energies are given in $\mathrm{MeV}$ per nucleon.

\begin{tabular}{lrrrrrrrr}
\hline \hline & CD-Bonn & ArV18 & Nijm1 & Nijm2 & \multicolumn{1}{c}{$A$} & $B$ & \multicolumn{1}{c}{$C$} & Reid \\
\hline$\langle E\rangle$ & -17.11 & -15.85 & -15.82 & -13.93 & -16.32 & -15.32 & -14.40 & -12.47 \\
$\langle V\rangle$ & -53.34 & -62.92 & -55.08 & -61.94 & -52.44 & -53.03 & -54.95 & -61.51 \\
$\langle T\rangle$ & 36.23 & 47.07 & 39.26 & 48.01 & 36.12 & 37.71 & 40.55 & 49.04 \\
$\left\langle V_{\pi}\right\rangle$ & -22.30 & -40.35 & -28.98 & -28.97 & -12.48 & -26.87 & -45.74 & -27.37 \\
$\langle E\rangle_{\mathrm{HF}}$ & 4.64 & 30.34 & 12.08 & 36.871 & 7.02 & 10.07 & 29.56 & 176.25 \\
$P_{D}(\%)$ & 4.83 & 5.78 & 5.66 & 5.64 & 4.38 & 4.99 & 5.62 & 6.47 \\
\hline \hline
\end{tabular}

the case of CD-Bonn to $36.9 \mathrm{MeV}$ for Nijm2, rather similar results are obtained in the BHF approximations. This demonstrates that the effect of correlations is quite different for the different interactions considered. However, it is worth noting that all these modern interactions are much "softer" than, e.g., the old Reid soft-core potential [12] in the sense that the HF result obtained for the Reid potential $(176 \mathrm{MeV})$ is much more repulsive.

Another measure for the correlations is the enhancement of the kinetic energy calculated for the correlated wave function as compared to the mean-field result which is identical to $T_{\mathrm{FG}}$, the energy per particle of the free Fermi gas. At the empirical density this value for $T_{\mathrm{FG}}$ is $23 \mathrm{MeV}$ per nucleon. One finds that correlations yield an enhancement for this by a factor which ranges from 1.57 in the case of CD-Bonn to 2.09 for Nijm1. It is remarkable that the effects of correlations, measured in terms of the enhancement of the kinetic energy or looking at the difference between the HF and BHF energies, are significantly smaller for the interactions CDBonn and Nijm1, which contain nonlocal terms.

Table I also lists the expectation value for the pionexchange contribution $V_{\pi}$ to the two-body interaction. Here one should note that the expectation value of $V_{\pi}$ calculated in the HF approximation is about $15 \mathrm{MeV}$ almost independent of the interaction considered. So it is repulsive and completely due to the Fock exchange term. If, however, the expectation value for $V_{\pi}$ is evaluated for the correlated wave function, one obtains rather attractive contributions ranging from $-22.30 \mathrm{MeV}$ per nucleon (CD-Bonn) to $-40.35 \mathrm{MeV}$ (ArV18). This expectation value is correlated to the strength of the tensor force or the $D$-state probability $P_{D}$ calculated for the deuteron (see Table I as well). Interactions with larger $P_{D}$, like the ArV18, yield larger values for $\left\langle V_{\pi}\right\rangle$. For a further support of this argument we also give the results for three different version of charge-independent Bonn potentials $A, B$, and $C$, defined in [11].

All this demonstrates that pionic and tensor correlations are very important to describe the binding properties of nuclei. In fact, the gain in binding energy due to correlations from $V_{\pi}$ alone is almost sufficient to explain the difference between the $\mathrm{HF}$ and BHF energies.

Until now we have just discussed results for nuclear matter at one density. The values for the kinetic energy $\langle T\rangle$, and $\left\langle V_{\pi}\right\rangle$ are displayed for various densities in Fig. 1. One finds that the ratio of the kinetic energy calculated for the correlated wave function, $\langle T\rangle$, and the energy of the free Fermi

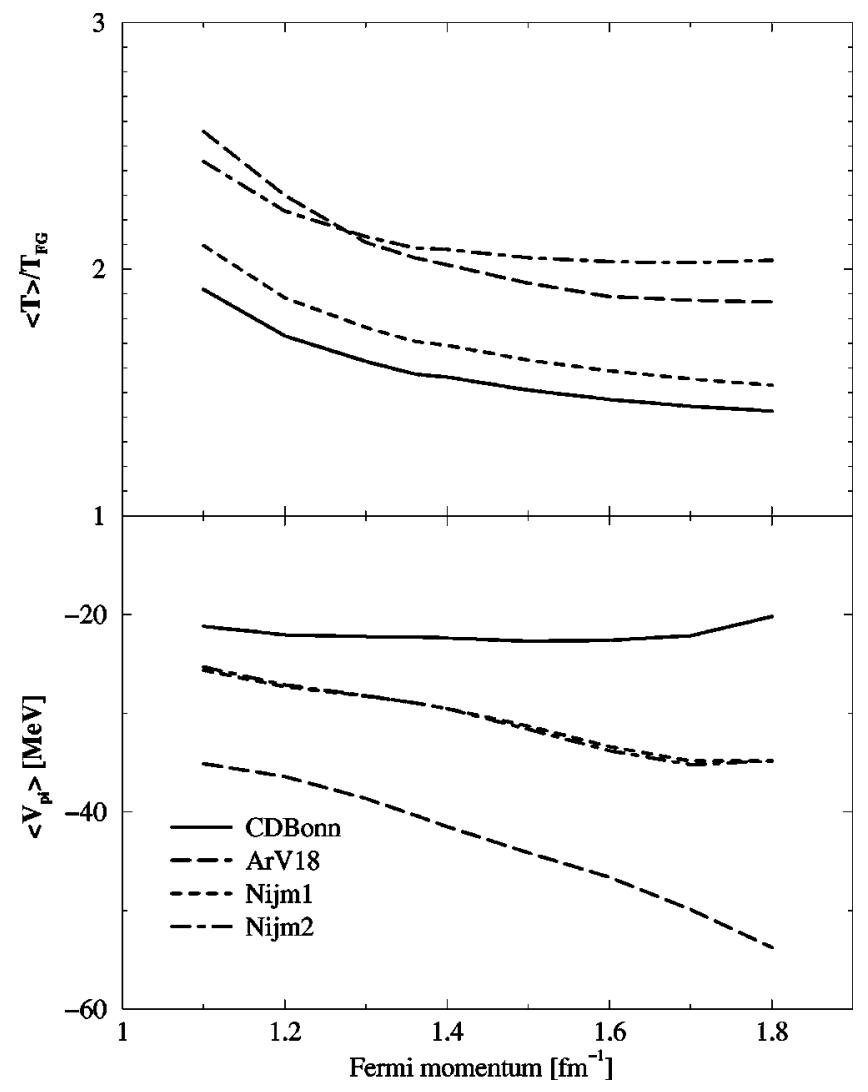

FIG. 1. The upper part of this figure displays the ratio of the kinetic energy per nucleon, calculated for the correlated state, to the energy per nucleon of a free Fermi gas (3) as a function of the Fermi momentum. The lower part exhibits the expectation value of the one-pion-exchange contribution to the binding energy per nucleon. Different realistic $N N$ interactions are considered. 


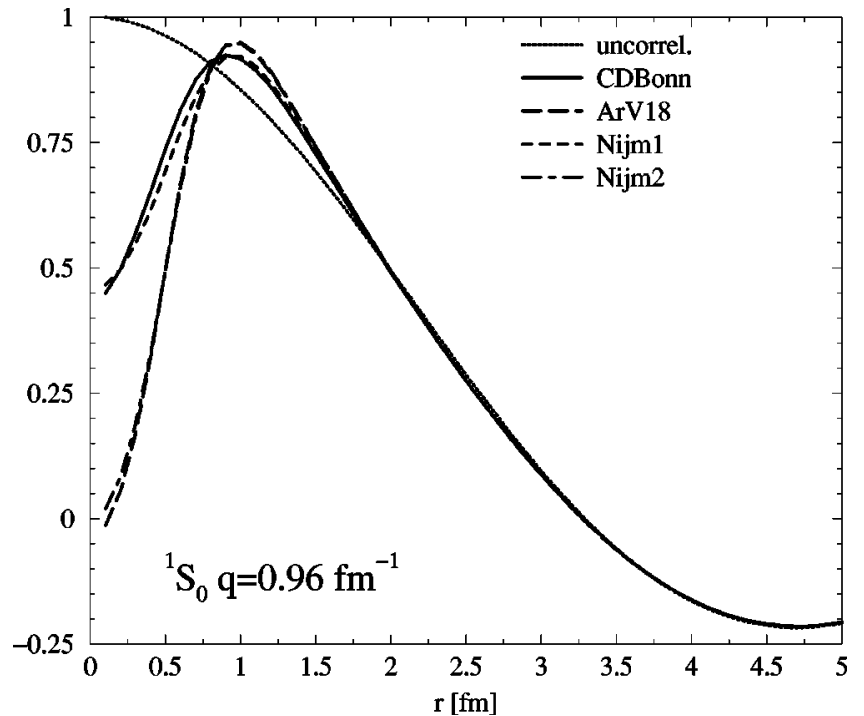

FIG. 2. Correlated wave functions $|i j\rangle_{\text {corr }}$ as defined in Eq. (5) as a function of the relative distance for the ${ }^{1} S_{0}$ partial wave. Results are shown for a pair of nucleons in nuclear matter at empirical saturation density, which heal to an uncorrelated two-nucleon wave function with momentum $q=0.96 \mathrm{fm}^{-1}$ at larger distances. The curves are labeled by the interactions, which were considered.

gas $\left\langle T_{\mathrm{FG}}\right\rangle$ decreases as a function of density. This plot furthermore shows that the results for the different interactions can be separated in two groups: the local interactions, ArV18 and Nijm2, yield larger kinetic energies than CD-Bonn and Nijm1, which contain nonlocal terms.

The lower part of Fig. 1 shows that the pionic contribution to the total energy is quite different for the interactions. It is strongest for ArV18, getting more attractive for larger densities. The pionic contribution obtained from the other potentials is weaker and does not exhibit this increase at high densities. This may indicate that the enhancement of pionic correlations, which has been discussed in the literature as an indication for pion condensation [13], is a feature which may not be reproduced by realistic interactions different from the Argonne potentials.

A different point of view on nuclear correlations may be obtained from inspecting the the relative wave functions for a correlated pair $|i j\rangle_{\text {corr }}$ defined in Eq. (5). Results for such correlated wave functions for a pair of nucleons in nuclear matter at empirical saturation density are displayed in Figs. 2 and 3. As an example we consider wave functions which "heal" at larger relative distances to an uncorrelated twonucleon wave function with momentum $q=0.96 \mathrm{fm}^{-1}$ calculated at a corresponding average value for the starting energy.

Figure 2 shows relative wave functions for the partial wave ${ }^{1} S_{0}$. One observes the typical features: a reduction of the amplitude as compared to the uncorrelated wave function for relative smaller than $0.5 \mathrm{fm}$, reflecting the repulsive core of the $N N$ interaction, an enhancement for distances between $\approx 0.7$ and $1.7 \mathrm{fm}$, which is due to the attractive components at medium range, and the healing to the uncorrelated wave function at large $r$. One finds that the reduction at short distances is much weaker for the interactions CD-Bonn and

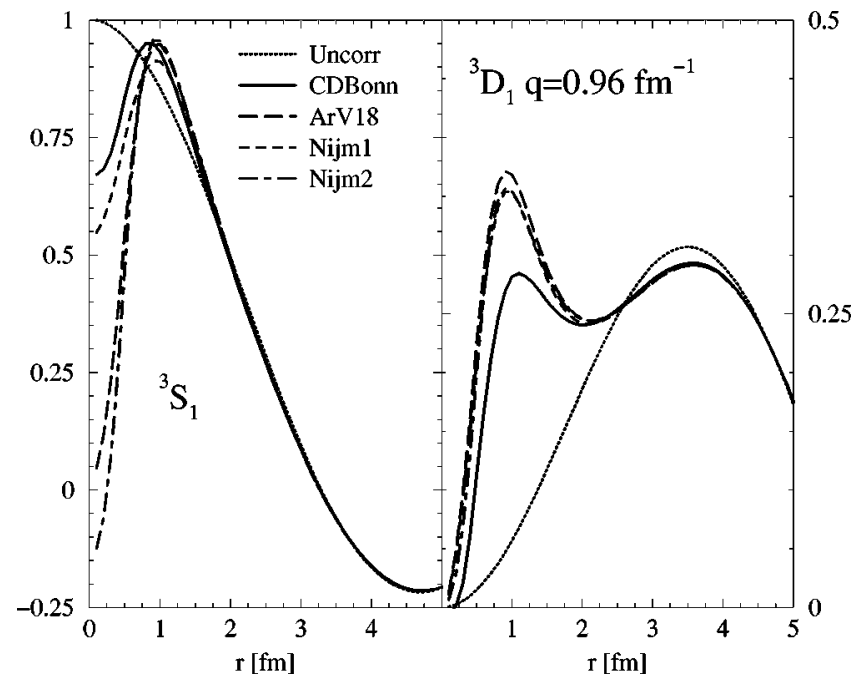

FIG. 3. Correlated wave functions as a function of the relative distance for the ${ }^{3} S_{1}$ and ${ }^{3} D_{1}$ partial waves. For further details, see Fig. 2.

Nijm1 than for the other two. This is in agreement with the discussion of the kinetic energies (see Fig. 1) and the difference between HF and BHF energies (see Table I). The nonlocal interactions CD-Bonn and Nijm1 are able to fit the $N N$ scattering phase shifts with a softer central core than the local interactions.

Very similar features are also observed in the ${ }^{3} S_{1}$ partial wave displayed in the left half of Fig. 3 . For the ${ }^{3} D_{1}$ partial wave, shown in the right part of Fig. 3, one observes a different behavior: All $N N$ interactions yield an enhancement of the correlated wave function at $r \approx 1 \mathrm{fm}$. This enhancement is due to the tensor correlations, which couples the partial waves ${ }^{3} S_{1}$ and ${ }^{3} D_{1}$. This enhancement is stronger for the interactions ArV18, Nijm1, and Nijm2 than for the CD-Bonn potential. Note that the former potential contain a pure nonrelativistic, local one-pion-exchange term, while the $\mathrm{CD}$ -

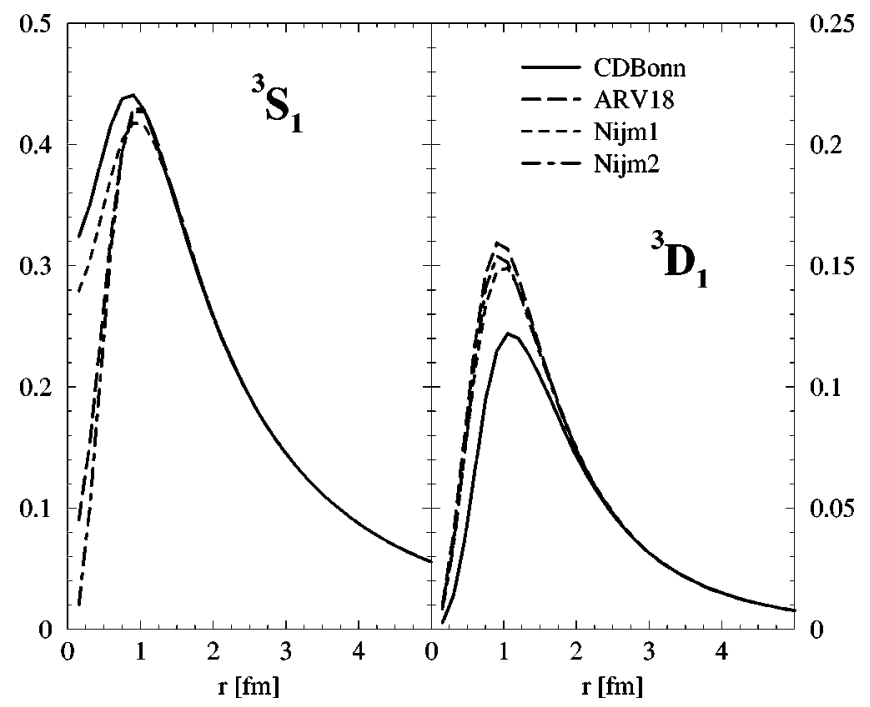

FIG. 4. Wave function for the deuteron $\left({ }^{3} S_{1}\right.$ and $\left.{ }^{3} D_{1}\right)$ calculated for different realistic interactions. 
Bonn contains a relativistic, nonlocal pion-exchange contribution. This behavior in the coupled ${ }^{3} S_{1}$ and ${ }^{3} D_{1}$ waves can also be observed in the corresponding wave functions for the deuteron, plotted in Fig. 4.

\section{CONCLUSIONS}

Four modern $N N$ interactions, the charge-dependent Bonn potential (CD-Bonn), the Argonne V18 (ArV18), and two versions of the Nijmegen potential (Nijm1 and Nijm2), which all give an excellent fit to $N N$ scattering phase shifts, exhibit significant differences in calculating $N N$ correlation functions and other observables in nuclear matter. Two of these interactions, CD-Bonn and Nijm1, contain nonlocal terms. These two interactions are considerably softer than the other interactions. This conclusion can be derived from three different observations: The Hartree-Fock energies are less repulsive, the kinetic energies calculated with the correlated wave functions are smaller and the correlated wave function in relative $S$ states are less suppressed at small relative distances.

The interactions also differ quite significantly in the pi- onic or tensor correlations they induce. This is indicated to some extent by the deuteron wave function, in particular by the $D$-state probability. These differences, however, are even enhanced in the nuclear wave functions leading to drastic differences in the pionic contribution to the nuclear binding energy. The Argonne potential in particular yields a large pionic contribution, which increases with density. This importance of the pionic correlations is not observed for the other interactions.

It would be of great interest to study whether the differences between the correlations predicted from these interactions can be observed in experiments like the exclusive $\left(e, e^{6} N N\right)$ reactions in order to discriminate the various models for the $N N$ interaction.

\section{ACKNOWLEDGMENTS}

This work was supported in part by the SFB 382 of the Deutsche Forschungsgemeinschaft, the DGICYT (Spain) Grant No. PB95-1249 and the Program SGR98-11 from Generalitat de Catalunya.
[1] C. J. G. Onderwater et al., Phys. Rev. Lett. 81, 2213 (1998).

[2] G. Rosner, Proceedings on Perspectives in Hadron Physics, edited by S. Boffi, C. Cioffi degli Atti, and M. Giannini (World Scientific, Singapore, 1998), p. 185.

[3] V. G. J. Stoks, R. A. M. Klomp, C. P. F. Terheggen, and J. J. de Swart, Phys. Rev. C 49, 2950 (1994).

[4] R. B. Wiringa, V. G. J. Stoks, and R. Schiavilla, Phys. Rev. C 51, 38 (1995).

[5] R. Machleidt, F. Sammarruca, and Y. Song, Phys. Rev. C 53, R1483 (1996).

[6] V. G. J. Stoks, R. A. M. Klomp, M. C. M. Rentmeester, and J.
J. de Swart, Phys. Rev. C 48, 792 (1993).

[7] A. Polls, H. Müther, R. Machleidt, and M. Hjorth-Jensen, Phys. Lett. B 432, 1 (1998).

[8] J. L. Forest, nucl-th/9905063.

[9] J. I. Musher, Am. J. Phys. 34, 267 (1966).

[10] M. I. Haftel and F. Tabakin, Nucl. Phys. A158, 1 (1970).

[11] R. Machleidt, Adv. Nucl. Phys. 19, 189 (1989).

[12] R. V. Reid, Jr., Ann. Phys. (N.Y.) 50, 411 (1968).

[13] A. Akmal and V. R. Pandharipande, Phys. Rev. C 56, 2261 (1997). 